\title{
FRAÇÕES DE ZINCO EM SOLO ARENOSO E SUAS RELAÇÕES COM DISPONIBILIDADE PARA Cynodon spp CV. TIFTON-85(1)
}

\author{
E. M. ANDRÉ(2), M. C. P. CRUZ(3), \\ M. E. FERREIRA(4) \& L. A. S. PALMA ${ }^{(5)}$
}

\begin{abstract}
RESUMO
Para avaliar um esquema de fracionamento de zinco em Argissolo arenoso e suas relações com a disponibilidade de $\mathrm{Zn}$ para Cynodon spp cv. Tifton-85, realizou-se um experimento em casa de vegetação, em esquema fatorial $5 \times 2 \times 2$ ( 5 doses de $Z n, 2$ doses de calcário e 2 épocas de amostragem), em delineamento inteiramente casualizado, com três repetições. As doses de $\mathrm{Zn}$ foram de 0; 2,5; 5,$0 ; 7,5$ e $10 \mathrm{mg} \mathrm{dm}^{-3}$; metade dos vasos não recebeu calagem ( $\left.\mathrm{V}=42 \%\right)$ e metade recebeu a calagem com vistas em elevar o índice de saturação por bases a $70 \%$. As épocas de amostragem foram 30 e 150 dias após aplicação de $Z n$, respectivamente, antes do plantio e depois do $3^{\circ}$ corte de Tifton-85. A aplicação de Zn resultou em aumento significativo do elemento nas frações: trocável, óxidos de Mn, matéria orgânica e óxidos de Fe. O Zn ligado aos óxidos de Mn aumentou significativamente com a calagem. Após $\mathbf{1 5 0}$ dias de experimentação, houve diminuição do $\mathrm{Zn}$ trocável, ligado aos óxidos de $\mathrm{Mn}$, à matéria orgânica e aos óxidos de $\mathrm{Fe}$, e aumento na fração residual. A distribuição de $\mathrm{Zn}$ nas frações do solo foi : residual >óxidos de $\mathrm{Fe}>$ óxidos de $\mathrm{Mn}$ >trocável >matéria orgânica. As relações entre as características estudadas mostraram que tanto o Zn-DTPA quanto o Zn trocável, ligado à M.O. e aos óxidos de Mn, foram eficientes para representar o Zn absorvido pela planta.
\end{abstract}

Termos para indexação: extração seqüencial, fracionamento, calagem, tifton.

\footnotetext{
(1) Trabalho apresentado no XXVIII CBCS (01 a 06/07/2001) em Londrina (PR). Recebido para publicação em dezembro de 2001 e aprovado em março de 2003.

(2) Doutoranda em Produção Vegetal pela Universidade Estadual Paulista - FCAV/UNESP. Via de acesso Prof. Paulo Donato Castellane, s/no. CEP 14884-900 J aboticabal (SP). Bolsista da FAPESP. E-mail: mangili @fcav.unesp.br

(3) Professora do Departamento de Solos e Adubos, FCAV/UNESP. E-mail: mcpcruz@fcav.unesp.br

(4) Professor Titular do Departamento de Solos e Adubos, FCAV/UNESP. E-mail: evaristo@fcav.unesp.br

(5) Zootecnista. Rua 10, 1333 CEP 14620-000 Orlândia (SP)
} 


\title{
SUMMARY: ZINC FRACTIONS IN A SANDY SOIL AND ITS RELATIONS WITH AVAILABILITY TO Cynodon spp CV. TIFTON-85
}

\begin{abstract}
To evaluate a fractionation schemefor $\mathrm{Zn}$ in a sandy soil (UItisol) and its relation with Zn availability for Cynodon spp cv. Tifton-85, an experiment was carried out in greenhouse, with a factorial scheme (5 doses of zinc, 2 doses of lime and 2 times of sampling), in a completely randomized block design, with thre replications. The doses of Zn were: 0; 2.5; 5.0; 7.5 and $10 \mathrm{mg} \mathrm{dm}^{-3}$; half of the pots did not received liming ( $\left.\mathrm{V}=42 \%\right)$ and, in theother half, liming was done to increase the base saturation index to $70 \%$. The sampling times were 30 and 150 days after Zn application, before planting and after cut of Tifton-85, respectively. Zinc application increased Zn in the exchangeable, associated to manganese oxides, associated to organic matter and associated to iron oxidefractions. Zinc associated with manganese oxides increased with liming. After 150 days of experimentation exchangeable zinc, Zn associated with manganese oxides, with organic matter and with iron oxides decreased, while residual Zn increased with the time. Zinc distribution was: residual >iron oxides > manganeseoxides $>$ exchangeable $>$ organic matter. The relations among thecharacteristics showed that DTPA-Zn and exchangeablezinc, Zn associated with organic matter, and $\mathrm{Zn}$ associated with manganese oxides were efficient to represent the zinc uptake by plants.
\end{abstract}

Index terms: sequential extraction, fractionation, liming, tifton.

\section{INTRODUÇÃO}

A disponibilidade de micronutrientes às plantas, incluindo a dos metais pesados, é influenciada por muitos fatores, sendo o pH do solo um dos mais importantes para el ementos como o Zn (Abreu et al., 2001). O efeito do pH na distribuição dos metais, em suas diferentes formas no solo, tem implicações importantes na retenção e mobilidade desses elementos, principalmente em solos contaminados (Sims, 1986; Zhu \& Alva, 1993).

Quando o pH do solo aumenta, o $\mathrm{Zn}$ é mais adsorvido pelos óxidos e hidróxidos de $\mathrm{Fe}$, de $\mathrm{Al}$ (Cavallaro \& McBride, 1984; Okazaki et al., 1986; Stahl \& J ames, 1991a) e de manganês (Stahl \& J ames, 1991b). Sims (1986), trabalhando com solo arenoso com baixo teor de matéria orgânica (1,6\%), verificou que, numa variação de $\mathrm{pH}$ de 4,8 a 7,7, o Zn trocável diminuiu de 42 para $1 \%$, enquanto o Zn ligado aos óxidos de Fe amorfos aumentou de 4 para $26 \%$, respectivamente. Zhu \& Alva (1993) observaram que a forma solúvel de $Z n$ foi $<2 \%$ em solos arenosos com pH 6,0 ou maior. Segundo Zhang et al. (1997), o aumento dopH dosolo causa a precipitação dos cátions metálicos sobre óxidos de Mn e de Fe cristalinos e amorfos, decrescendo, por sua vez, a forma trocável e a ligada à matéria orgânica. A acidificação gradual do solo, principal mente devida à lixiviação, também leva a mudanças nas formas dos metais.

Apesar da influência do pH do solo na adsorção dos metais, Amaral Sobrinho et al. (1993) comentam que os el ementos relativamente móveis, como o Zn, encontram-se, predominantemente, nas frações: solúvel, trocável eassociada a carbonatos, enquanto os de baixa mobilidade estão ligados às frações: óxidos de Fe e de $\mathrm{Mn}$, orgânica e residual.

Os métodos de extração seqüencial são importantes para melhorar a compreensão a respeito de quais frações estariam disponibilizando os micronutrientes às plantas (Shuman, 1986). Vários deles também têm si do utilizados em estudos de contaminação do solo com metais pesados, dentre os quais se destacam os de Shuman (1985), Tessier et al. (1979), Sposito et al. (1982) e Miller et al. (1986). As princi pais limitações desses métodos são: a falta de sel etividade da mai oria dos extratores por determinada fração; o potencial para readsorção dos elementos por componentes do solo durante a extração; outras variáveis, como, por exemplo, a secagem da amostra de solo, incluídas no processo de extração. Em resposta a essas limitações, podese dizer que tais métodos oferecem a mel hor medida das associações químicas entre os elementos em estudo e as formas amorfas e cristal inas do solo, que não são facilmente avaliadas por outros tipos de medida (Amacher, 1996).

O objetivo do presentetrabalho foi o dequantificar o Zn presente em diferentes frações em sol o arenoso, submetido ou não à calagem, eaval iar suas rel ações com o Zn extraído com DTPA e o Zn disponível para Cynodon spp cv. Tifton-85.

\section{MATERIAL E MÉTODOS}

O ensaio foi realizado em casa de vegetação, empregando esquema fatorial $5 \times 2 \times 2$ (cinco doses 
de $\mathrm{Zn}$, duas doses de calcário e duas épocas de amostragem), em delineamento inteiramente ao acaso, com três repetições, totalizando 30 vasos.

O solo utilizado refere-se ao horizonte $\mathrm{A}$ de um Argissolo Vermelho-Amarelo distrófico, com $850 \mathrm{~g} \mathrm{~kg}^{-1}$ de areia, 80 de silte e 70 de argila. Foi coletado em Taquaritinga (SP) na camada de 0 $20 \mathrm{~cm}$, seco, destorroado, peneirado $(6 \mathrm{~mm}$ de abertura de malha), homogeneizado e amostrado. $\mathrm{Na}$ amostra, foram feitas análises de $\mathrm{pH}$ em $\mathrm{CaCl}_{2}$, $\mathrm{P}$ (resina), $\mathrm{K}, \mathrm{Ca}, \mathrm{Mg}, \mathrm{M} . \mathrm{O} ., \mathrm{H}+\mathrm{Al}$ e $\mathrm{Al}$, segundo Raij et al . (1987); S-SO ${ }_{4}^{2-}$, segundo Cantarella \& Raij (1997); Cu, Fe, Mn e Zn extraídos com DTPA pH 7,3, segundo Lindsay \& Norvell (1978). Os resultados obtidos foram: $\mathrm{pH}^{-m} \mathrm{CaCl}_{2}=4,6$; $\mathrm{P}$ resina $=3 \mathrm{mg} \mathrm{dm}^{-3}$; M.O.=17 g dm${ }^{-3} ; \mathrm{K}$; Ca; Mg; $\mathrm{H}+\mathrm{Al}$; $\mathrm{Al}$; SB e CTC, em mmol ${ }_{\mathrm{C}} \mathrm{dm}^{-3}$, respectivamente, 2,$1 ; 8 ; 6 ; 22 ; 2 ; 16 \mathrm{e}$ 38; $\mathrm{V}=42 \%$; S-SO ${ }_{4}^{2-;} \mathrm{Cu} ; \mathrm{Fe} ; \mathrm{Mn}$ e Zn, em mg dm³ respectivamente, 1; 0,5; 23; 30,0 e 0,4.

Em metade dos tratamentos, o solo foi mantido em condição original de $\mathrm{pH}$ e, na outra metade, a calagem foi feita utilizando $\mathrm{CaCO}_{3}$ e $\mathrm{MgCO}_{3}$ p.a. (Ca:Mg =4:1), demodo a elevar oíndice de saturação por bases a $70 \%$. Caixas de plástico com $12 \mathrm{~cm}$ de altura, $43 \mathrm{~cm}$ de comprimento e $28 \mathrm{~cm}$ de largura, dotadas de dreno, foram empregadas para a incubação do equivalente a $10,5 \mathrm{dm}^{3}$ de solo, por 30 dias. A umidade foi mantida acerca de $60 \%$ da sua capacidade de retenção de água por meio de pesagens, com correção do peso pela adição de água deionizada.

Cinco dias após o início do período de incubação com cal cário, foi aplicada solução de $\mathrm{ZnSO}_{4} \cdot 7 \mathrm{H}_{2} \mathrm{O}$, de modo a adicionar $0\left(Z n_{0}\right), 2,5\left(Z n_{1}\right), 5,0\left(Z n_{2}\right), 7,5$ $\left(Z n_{3}\right)$ e $10,0 \mathrm{mg} \mathrm{dm}^{-3}\left(Z_{4}\right)$ de $Z n$. Dez dias após a adição de $Z n$, foi aplicada, superficialmente, uma solução de nutrientes de modo a adicionar $100 \mathrm{mg} \mathrm{dm}^{-3}$ de N, 100 deP, 100 deK, 20 deS, 0,5 de $\mathrm{B}, 1,25$ de $\mathrm{Cu}, 3,0$ de $\mathrm{Mn}$ e 0,02 de Mo. As fontes empregadas foram: $\mathrm{NH}_{4} \mathrm{NO}_{3}, \mathrm{NH}_{4} \mathrm{H}_{2} \mathrm{PO}_{4}, \mathrm{KNO}_{3}$, $\mathrm{KH}_{2} \mathrm{PO}_{4}, \mathrm{~K}_{2} \mathrm{SO}_{4}, \mathrm{H}_{3} \mathrm{BO}_{3}, \mathrm{CuSO}_{4} \cdot 5 \mathrm{H}_{2} \mathrm{O}, \mathrm{MnCl}_{2} .2 \mathrm{H}_{2} \mathrm{O}$ $\mathrm{e}\left(\mathrm{NH}_{4}\right)_{6} \mathrm{M} \mathrm{O}_{7} \mathrm{O}_{24} \cdot 4 \mathrm{H}_{2} \mathrm{O}$.

Após a incubação com calcário, Zn e demais fertilizantes, osol ofoi seco, peneirado, homogeneizado eamostrado. As amostras foram de cerca de $0,5 \mathrm{dm}^{3}$ e nelas foram feitas determinações de $\mathrm{pH}, \mathrm{Zn}$ total, Zn extraído por DTPA e das frações de $Z n$.

O solo restante $\left(10 \mathrm{dm}^{3}\right)$ foi devolvido para as caixas de plástico e, em cada uma delas, foram plantados seis estolões de Cynodon spp cv. Tifton85 , cada um com 3 a 4 gemas. Treze dias após o transplantio das mudas, foi realizado um corte a $10 \mathrm{~cm}$ do solo para uniformizar as plantas e, posteriormente, foram feitos três cortes, aos 45,75 e 120 dias do plantio. Até o primeiro corte, foram adicionados mais $145 \mathrm{mg} \mathrm{dm}^{-3}$ de $\mathrm{N}$ e 120 de K ; entre o primeiro e o segundo corte, foram adicionados $170 \mathrm{mg} \mathrm{dm}^{-3}$ de N edeK e20 deS; e entreo segundo e o terceiro corte, foram adicionados $280 \mathrm{mg} \mathrm{dm}^{-3}$ de $\mathrm{N}$ e deK, 30 deP , 20 de $\mathrm{S}$ e 1 de $\mathrm{Cu}$, pela aplicação de $\mathrm{NH}_{4} \mathrm{NO}_{3}, \mathrm{NH}_{4} \mathrm{H}_{2} \mathrm{PO}_{4}, \mathrm{KNO}_{3}, \mathrm{~K}_{2} \mathrm{SO}_{4}$ e $\mathrm{CuSO}_{4}$ total izando, em cobertura, $595 \mathrm{mg} \mathrm{dm}^{-3} \mathrm{de} \mathrm{N}, 570$ de $K, 30$ de $P, 40$ de $\mathrm{S}$ e 1 de $\mathrm{Cu}$.

Após cada corte, as plantas foram lavadas com solução de detergente $\left(1 \mathrm{~mL} \mathrm{~L}^{-1}\right)$ e duas vezes em água dei onizada, e secas em estufa a $65^{\circ} \mathrm{C}$ até peso constante para posterior obtenção da matéria seca. Obtida a produção, as plantas foram moídas e as amostras submetidas à digestão nítrico-perclórica para a determinação de Zn (Bataglia et al., 1983).

Ao final do experimento, as raízes foram eliminadas, a porção de solo de cada caixa foi seca, peneirada, e uma amostra foi coletada para a determinação do teor de $Z n$ total, de $Z n$ solúvel em DTPA e para o fracionamento de $Z n$.

O procedimento de fracionamento descrito a seguir teve como base os métodos propostos por Chao (1972), Shuman (1985), Camargo et al. (1986) e Miller et al. (1986), aos quais foram introduzidas modificações principal menteem relação à massa da amostra, à concentração da solução extratora para a fração trocável eao número de extrações da fração óxidos de Fe. As frações foram extraídas na ordem descrita a seguir, tendo sido a fração orgânica extraída posteriormente aos óxidos de $\mathrm{Mn}$, visto que, segundo Miller et al. (1986), a solução de $\mathrm{K}_{4} \mathrm{P}_{2} \mathrm{O}_{7}$ dissolve parcialmente os óxidos de $\mathrm{Mn}$, motivo pelo qual a extração deve ser ordenada para solubilizálos antes.

Zn trocável (Shuman, 1985): cerca de $5 \mathrm{~cm}^{3}$ de solo foram triturados em almofariz e passados por peneira $\mathrm{n} 060(0,25 \mathrm{~mm}$ de abertura de malha). Subamostras de 2,5 g foram colocadas em tubos de centrífuga com capacidade para $50 \mathrm{~mL}$. Em seguida, foram transferidos $20 \mathrm{~mL}$ de solução de cloreto de magnésio $\left[\mathrm{Mg}\left(\mathrm{NO}_{3}\right)_{2}\right] 1 \mathrm{~mol} \mathrm{~L}-1$ para cada tubo e procedeu-se à agitação em agitador horizontal a 140 rpm por $30 \mathrm{~min}$. O material foi então centrifugado por $10 \mathrm{~min}$ a $3.000 \mathrm{rpm}$, e o sobrenadantefoi filtrado e recebido em balão volumétrico com capacidade para $50 \mathrm{~mL}$. O sedimento foi ressuspendido com $10 \mathrm{~mL}$ de solução de nitrato de cálcio $\left[\mathrm{Ca}\left(\mathrm{NO}_{3}\right)_{2}\right]$ $0,01 \mathrm{~mol} \mathrm{~L}^{-1}$ e centrifugado novamente, enquanto 0 sobrenadante foi filtrado e recebido no mesmo balão volumétrico. Repetiu-se o procedimento de lavagem com solução de $\mathrm{Ca}\left(\mathrm{NO}_{3}\right)_{2}$ 0,01 mol L-1. Ao final, o sedimento (sedimento $A$ ) foi reservado para a etapa seguintee o balão volumétrico que recebeu os filtrados foi completado a $50 \mathrm{~mL}$ com água deionizada.

Zn ligado aos óxidos de manganês (Chao, 1972): $20 \mathrm{~mL}$ de solução de cloridrato de hidroxilamina $\left(\mathrm{NH}_{2} \mathrm{OH} . \mathrm{HCl}\right) 0,1 \mathrm{~mol} \mathrm{~L}^{-1}, \mathrm{pH} 2$, foram adicionados ao tubo de centrífuga que continha o sedimento A, que foi ressuspendido, e procedeu-se à agitação em agitador horizontal a $140 \mathrm{rpm}$ por $30 \mathrm{~min}$. O material foi centrifugado por $10 \mathrm{~min}$ a $3.000 \mathrm{rpm}$, sendo o sobrenadante filtrado e recebido em balão volumétrico com 
capacidade para $50 \mathrm{~mL}$. O sedimento foi lavado como já descrito para a fração trocável. O volume foi completado a $50 \mathrm{~mL}$ com água deionizada e o sedimento (sedimento $B$ ) reservado.

Zn ligado à matéria orgânica (Miller et al., 1986): $20 \mathrm{~mL}$ de solução de pirofosfato de potássio $\left(\mathrm{K}_{4} \mathrm{P}_{2} \mathrm{O}_{7}\right)$, $1 \mathrm{~mol} \mathrm{~L}^{-1}$ foram adicionados ao tubo de centrífuga que continha o sedimento $B$, que foi ressuspendido, e procedeu-se à agitação em agitador horizontal a $140 \mathrm{rpm}$ por $12 \mathrm{~h}$ (para solo argil oso ou com teores altos de matéria orgânica, o tempo deve ser de $24 \mathrm{~h}$ ). O material foi centrifugado por $10 \mathrm{~min}$ a $3.000 \mathrm{rpm}$, sendo o sobrenadante filtrado erecebi do em balão volumétrico com capacidade para $50 \mathrm{~mL}$. $\mathrm{O}$ procedimento para lavagem do sedimento foi 0 mesmojá descrito, eo volume foi completado a $50 \mathrm{~mL}$ com água deionizada. O sedimento (sedimento C) foi reservado.

Zn ligado aos óxidos de ferro (Shuman, 1985): $20 \mathrm{~mL}$ da solução que continha citrato neutro de amônio $\left(\mathrm{NH}_{4} \mathrm{C}_{2} \mathrm{O}_{4}\right)$ 0,2 mol L-1, ácido cítrico $\left(\mathrm{H}_{2} \mathrm{C}_{2} \mathrm{O}_{4}\right)$ $0,2 \mathrm{~mol} \mathrm{~L}^{-1}$ e ácido ascórbico $\left(\mathrm{C}_{6} \mathrm{H}_{8} \mathrm{O}_{6}\right) 0,1 \mathrm{~mol} \mathrm{~L}^{-1}$, $\mathrm{pH} 3$, foram adicionados ao tubo de centrífuga com o sedimento C, que foi ressuspendido. O material foi colocado em banho-maria por $30 \mathrm{~min}$, submetido à agitação ocasional, em seguida centrifugado por $10 \mathrm{~min}$ a $3.000 \mathrm{rpm}$, e o sobrenadante foi filtrado e recebi do em balão vol umétri co com capaci dade para $50 \mathrm{~mL}$. Foi feita mais uma extraçãosucessiva, sendo o sedimento final lavado uma vez com $10 \mathrm{~mL}$ de solução de $\mathrm{Ca}\left(\mathrm{NO}_{3}\right)_{2}$ 0,01 mol L-1, centrifugado, e o sobrenadante filtrado e recebido no mesmo balão volumétrico. O volume foi completado a $50 \mathrm{~mL}$ com água deionizada. Para outros tipos de solo, devem ser feitas extrações sucessivas até que o sedimento apresente cor cinza. O sedimento dessa extração foi seco em estufa acerca de $65^{\circ} \mathrm{C}$, pesado e triturado em al mofariz (sedimento D).

Zn residual (Camargo et al., 1986): em cadinhos de teflon, for am col ocados $0,5 \mathrm{~g}$ de sedimento $D$ seco e triturado, três gotas de água deionizada, $1 \mathrm{~mL}$ de ácido perclórico $\left(\mathrm{HClO}_{4}\right)$, tendo sido o material prédigerido a frio por uma noite. No outro dia, foram adicionados 7,5 mL de ácido fluorídrico (HF) à amostra, e o cadinho foi colocado em banho de areia até próximo à secagem. Os cadinhos foram resfriados, receberam mais 7,5 $\mathrm{mL}$ de HF elevados ao banho de areia, no qual ficaram até próximo à secagem. Os cadinhos foram resfriados novamente e, em seguida, após adição de $5 \mathrm{~mL}$ de solução de ácido clorídrico $(\mathrm{HCl}) 3 \mathrm{~mol} \mathrm{~L}^{-1}$ e $5 \mathrm{~mL}$ de água deionizada, colocados no banho de areia até à fervura. Depois de frio, o conteúdo de cada cadinho foi transferido para balão volumétrico de $25 \mathrm{~mL}$, e o volume foi completado com água deionizada. O resultado obtido nessa fração foi corrigido com base na relação massa de resíduo/massa de solo da amostra.
Todas as filtrações das frações, trocável, óxidos de $M n$, matéria orgânica eóxidos deFe, foram feitas em papel defiltroWhatman no 42, eas quantificações, em espectrofotômetro de absorção atômica. O Zn total do solo por extração direta foi feito da mesma maneira, como descrito para a fração residual.

Os dados obtidos foram submetidos à análise de variância pel oteste $F$, eas médias foram comparadas pelo teste de Tukey. Foram feitas as seguintes análises de regressão: (a) teor de Zn extraído por DTPA e quantidade de $Z n$ absorvido pela planta por vaso; (b) teor de zinco extraído por DTPA e Zn em cada fração no solo, e (c) quantidade de Zn absorvido pela planta eem cada fração no solo, visando prever em qual delas o elemento poderia ser considerado disponível.

\section{RESULTADOS E DISCUSSÃO}

Os dados obtidos apontam para a seguinte ordem de distribuição de Zn entreas frações, independentemente da época de amostragem: residual > ligado aos óxidos de $\mathrm{Fe}$ >ligado aos óxidos de $\mathrm{Mn}>$ >trocável > ligado à matéria orgânica (Quadro 1).

Em termos percentuais, a distribuição de $Z n$ em cada fração, em relação ao total obtido pela soma das frações, nas amostras de sol o col etadas antes do plantio do tifton, é de: 3 a 15 \% na fração trocável; 5 a $20 \%$ associados aos óxidos de $\mathrm{Mn} ; 2$ a 6 \% ligados à matéria orgânica; 24 a 35 \% ligados aos óxidos de Fe; e 35 a 57 \% na fração residual (Quadro 1). Esses valores assemel ham-se aos encontrados por Chowdhury et al. (1997), que trabalharam com 20 tipos de solo da Nova Zelândia, os quais receberam $20 \mathrm{mg} \mathrm{dm}^{-3}$ de $\mathrm{Zn}$ e apresentaram, em média, $3 \%$ do total de Zn na forma trocável; $5 \%$ ligados à matéria orgânica; 9, 18 e $24 \%$ associados aos óxidos de $\mathrm{Mn}$, de $\mathrm{Fe}$ amorfo e cristalino, respectivamente; e 40 \% na fração residual. É importante ressaltar que os valores encontrados referem-se a um sol o de textura arenosa; para sol os de textura argilosa, é possível que a distribuição de Zn seja diferente.

A quantidadede $Z n$ recuperada pel ofracionamento em relação à digestão perclórico-fluorídrica variou de 89 a $101 \%$, considerando ambas as amostragens, e o Zn aplicado, que foi recuperado pela digestão perclórico-fluorídrica, foi de $97 \%$, em média (Quadro 1). Sims \& Kline(1991) recuperaram 91 \% do $\mathrm{Zn}$ pelo fracionamento (soma das frações) em relação ao total por digestão com $\mathrm{HNO}_{3} 4 \mathrm{~mol} \mathrm{L-1}$. Davidson et al. (1994) obtiveram $96 \%$ de recuperação do $\mathrm{Zn}$ pelo fracionamento em relação ao total por digestão com $\mathrm{HCl}+\mathrm{HNO}_{3}$, em microondas, e Howard \& Vandenbrink (1999), trabalhando com sedimentos artificiais de composição variável, conseguiram recuperar de 80 
Quadro 1. Frações de zinco no solo antes do plantio e depois da colheita do tifton

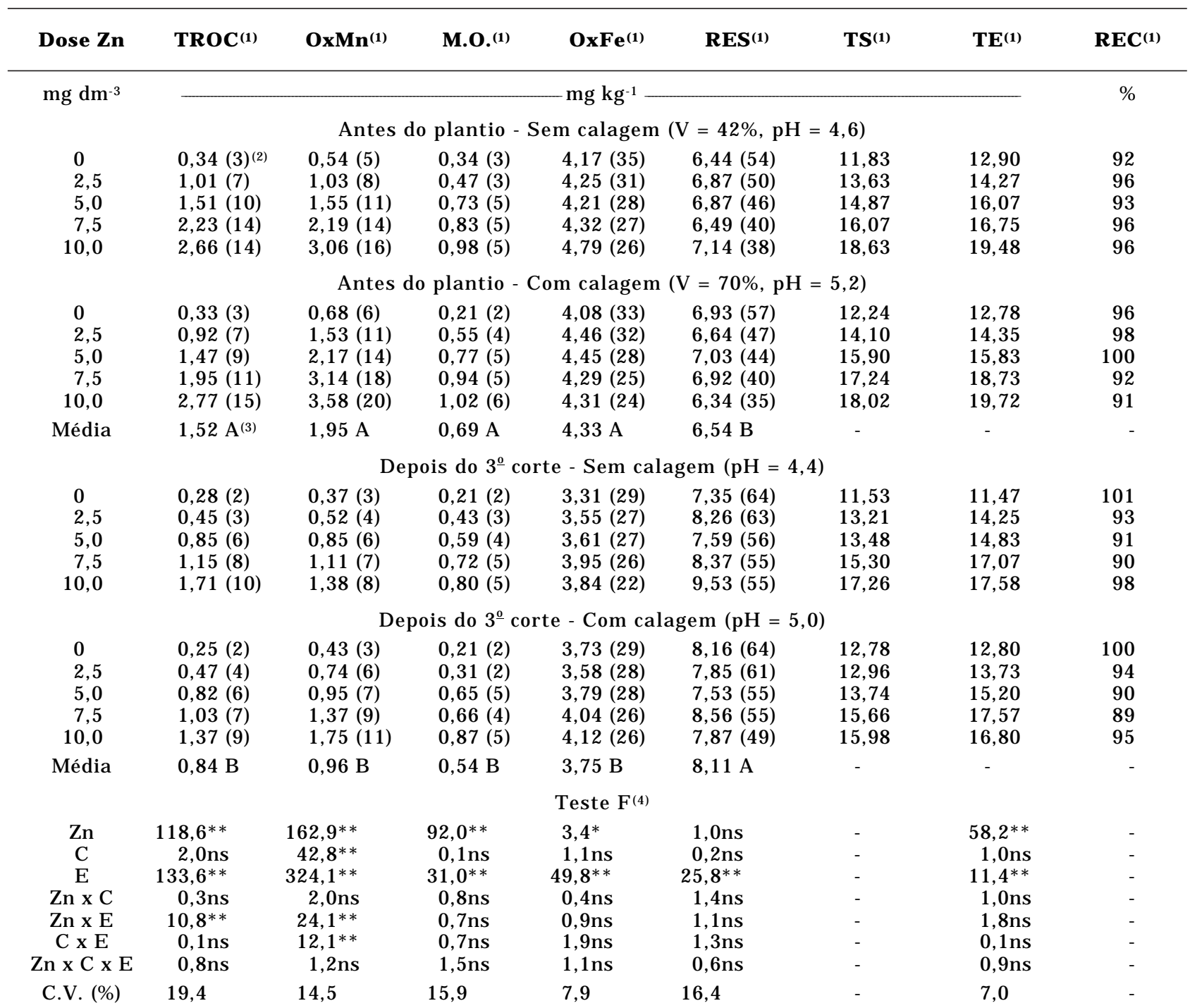

(1) Em queTROC =trocável; OxMn =ligado aos óxidos de Mn; M.O. =ligado à matéria orgânica; OxFe =ligado aos óxidos de Fe; RES = residual; TS = total por soma das frações; TE = total por extração direta; REC = (total por soma/total por extração)100. (2) Os valores entre parênteses, correspondem à percentagem da soma em cada fração. ${ }^{(3)}$ Médias seguidas de mesma letra não diferem

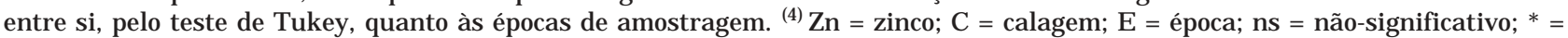
significativo a $5 \% \mathrm{e}^{* *}=$ significativo a $1 \%$, pelo teste $\mathrm{F}$.

a 99 \% do Zn pelo fracionamento em relação ao total medido. Segundo Shuman (1986), a soma das frações deveria ser igual ao total obtido pela digestão e as diferenças poderiam ocorrer pelo acúmulo de pequenos erros nas extrações das frações.

Houve efeito significativo da aplicação de Zn em todas as frações, exceto na residual e no $\mathrm{Zn}$ total (Quadro 1). Para valores absolutos, o efeito significativo da aplicação de Zn levou ao aumento na quantidade presentenas frações: trocável, ligado aos óxidos de $\mathrm{Mn}$, à matéria orgânica e aos óxidos de $\mathrm{Fe}$, mas não teve efeito na fração residual. Para o Zn nas frações (matéria orgânica e óxidos de Fe), os dados gerais e as anál ises de regressão apontam para o efeito linear da aplicação de Zn no aumento da quantidade do elemento nessas frações ( $\hat{y}=$ $\left.0,067 x+0,277, R^{2}=0,976 * *\right)$ e $(\hat{y}=0,043 x+3,828$ $\left.\mathrm{R}^{2}=0,988^{* *}\right)$, respectivamente.

Considerando os val ores percentuais, somenteas frações óxidos de Fe e residual foram diferentes, ou seja, houve diminuição com o aumento de $\mathrm{Zn}$ aplicado. I sso indica que, em relação ao Zn aplicado, as maiores quantidades permaneceram nas frações mais solúveis ou de mais fácil solubilização, e que o 
tempo de contato inicial do Zn com o solo (30 dias) foi insuficiente para permitir a adsorção específica de Zn nos óxidos de Feeminerais silicatados do solo.

A calagem aumentou significativamente a quantidade de $\mathrm{Zn}$ associado aos óxidos de $\mathrm{Mn}$ (Quadro 1). O aumento do $\mathrm{pH}$ favorece a adsorção de $\mathrm{Zn}$ aos óxidos de $\mathrm{Fe}$, Al e $\mathrm{Mn}$ do solo, nos quais são formados complexos de esfera interna com os cátions metálicos. Tal comportamento, chamado de adsorção específica ou quimiossorção, é citado por diversos autores, dentre os quais Amaral Sobrinho et al. (1997). O efeito da calagem não foi significativo para as demais frações, provavelmente pel o tipo de solo, de textura arenosa, com baixa atividade de argilas, pequeno teor de matéria orgânica eóxidos e pela pequena variação de $\mathrm{pH}$ do solo obtida.

Quanto à época de amostragem, o efeito significativo indica que houve redistribuição de Zn entre as frações, com diminuição do elemento associado às frações consideradas mais solúveis ou de mais fácil solubilização (trocável, ligado aos óxidos de $\mathrm{Mn}$, à matéria orgânica e aos óxidos de $\mathrm{Fe}$ ), e aumento do $\mathrm{Zn}$ residual após a colheita do tifton (Quadro 1). O tempo de contato do Zn com o solo (150 dias) permitiu a sua redistribuição para a fração residual. Essa fração é considerada como sendo formada principal mente por óxidos de Fe altamente cristal inos e minerais silicatados (Ma \& Uren, 1998). A migração do Zn para a fração mais estável (residual) ocorreu mesmo com a diminuição do pH do solo após o 3o corte do tifton (Quadros 1 e 2).

Não houve efeito significativo da interação $Z n x$ calagem, provavelmente pela pequena variação de pH observada (Quadro 2); percebeu-se, porém, efeito significativo da interação $\mathrm{Zn} x$ época nas frações óxidos de Mn e trocável (Quadros 1 e 3).
Os dados mostraram que, exceto para os tratamentos que não receberam Zn, o teor de Zn nas frações óxidos de $M n$ e trocável diminuiu de forma significativa da primeira para a segunda época de amostragem (Quadro 3).

À medida que aumentou a quantidade de $\mathrm{Zn}$ disponível no solo, a absorção pela planta foi maior, indicando que o DTPA mostrou-se eficiente para representar o comportamento da planta (Figura 1). Essa constatação confirma a característica atribuída ao extrator DTPA, relatada por diversos autores, de detectar o efeito do $\mathrm{pH}$ sobre o teor do elemento extraído do solo (Shuman, 1986; Abreu \& Raij, 1996; Oliveira et al., 1999). Shuman (1986) relatou que um extrator usado para micronutrientes, para ser sensível às mudanças de $\mathrm{pH}$ do solo e refletir a disponibilidade para a planta, deve remover, em parte ou totalmente, a fração trocável e, quanto ao Zn e Mn, não remover a fração orgânica. Ainda, comenta o autor que um extrator fortemente ácido em contato com o solo, por tempo longo, também pode remover parte da fração oxídica.

O conhecimento das diferentes frações de um nutriente no solo permite que se associe cada uma del as com oteor obtido por um extrator quími co usado para avaliar a disponibilidade do nutriente em questão, bem como com o absorvido pel a planta, dando mai or consistência às relações encontradas. Assim, à vista da relação significativa entre o $\mathrm{Zn}$ do solo extraído por DTPA e o absorvido pela planta, foram feitas equações de regressão entre o Zn-DTPA e o el emento em cada fração, antes do plantio (Figura 2) e depois do 3 o corte de tifton (Figura 3), e entre $Z n$ em cada fração e o Zn na parte aérea da planta, antes do plantio (Figura 4) e depois do 3을 corte de tifton (Figura 5), na tentativa de prever (Figuras 2 e 4 ) e

Quadro 2. Valores de pH e teores de zinco extraído por DTPA em amostras de solo coletadas antes do plantio e depois do $3^{\circ}$ corte de tifton e de $\mathrm{Zn}$ na parte aérea de plantas

\begin{tabular}{|c|c|c|c|c|c|c|}
\hline \multirow{2}{*}{ Dose de Zn } & \multicolumn{2}{|c|}{ Antes do plantio } & \multicolumn{2}{|c|}{ Depois do $3^{\circ}$ corte } & \multicolumn{2}{|c|}{ Zn na planta } \\
\hline & pH em $\mathrm{CaCl}_{2}$ & Zn-DTPA & $\mathrm{pH}$ em $\mathrm{CaCl}_{2}$ & Zn-DTPA & $1^{\circ}$ corte & $3^{\circ}$ corte \\
\hline $\mathrm{mg} \mathrm{dm} \mathrm{dm}^{-3}$ & & $\mathrm{mg} \mathrm{dm}-3$ & & $\mathrm{mg} \mathrm{dm} \mathrm{dm}^{-3}$ & \multicolumn{2}{|c|}{ mg vaso-1 } \\
\hline $\begin{array}{r}0 \\
2,5 \\
5,0 \\
7,5 \\
10,0\end{array}$ & $\begin{array}{l}4,6 \\
4,6 \\
4,6 \\
4,6 \\
4,6\end{array}$ & $\begin{array}{l}0,4 \\
2,2 \\
4,0 \\
5,9 \\
8,5\end{array}$ & $\begin{array}{c}\text { Sem calagem } \\
4,4 \\
4,4 \\
4,3 \\
4,4 \\
4,4\end{array}$ & $\begin{array}{l}0,2 \\
0,9 \\
2,1 \\
3,0 \\
4,2\end{array}$ & $\begin{array}{l}1,51 \\
2,68 \\
4,10 \\
4,68 \\
5,12\end{array}$ & $\begin{array}{l}1,08 \\
3,17 \\
4,61 \\
5,92 \\
7,24\end{array}$ \\
\hline \multicolumn{7}{|c|}{ Com calagem } \\
\hline $\begin{array}{r}0 \\
2,5 \\
5,0 \\
7,5 \\
10,0\end{array}$ & $\begin{array}{l}5,2 \\
5,2 \\
5,2 \\
5,2 \\
5,2\end{array}$ & $\begin{array}{l}0,3 \\
1,9 \\
3,7 \\
5,8 \\
8,1\end{array}$ & $\begin{array}{l}4,9 \\
5,0 \\
4,9 \\
5,0 \\
4,9\end{array}$ & $\begin{array}{l}0,2 \\
0,9 \\
1,8 \\
2,8 \\
3,8\end{array}$ & $\begin{array}{l}1,18 \\
2,75 \\
3,55 \\
4,73 \\
5,06\end{array}$ & $\begin{array}{l}1,02 \\
2,97 \\
4,65 \\
5,97 \\
7,20\end{array}$ \\
\hline
\end{tabular}


Quadro 3. Teores de zinco trocável e ligado aos óxidos de Mn, na interação entre doses do elemento e épocas de amostragem

\begin{tabular}{|c|c|c|c|c|c|}
\hline \multirow{2}{*}{ É poca de amostragem } & \multicolumn{5}{|c|}{ Dose de $\mathrm{Zn}\left(\mathrm{mg} \mathrm{dm}^{-3}\right)$} \\
\hline & $\mathbf{0}$ & 2,5 & 5,0 & 7,5 & 10,0 \\
\hline Dia & \multicolumn{5}{|c|}{ Zn trocável, mg dm-3 } \\
\hline $\begin{array}{r}30 \\
150\end{array}$ & $\begin{array}{l}0,33 \mathrm{Ae}^{(1)} \\
0,26 \mathrm{Ac}\end{array}$ & $\begin{array}{l}0,97 \mathrm{Ad} \\
0,46 \mathrm{BC}\end{array}$ & $\begin{array}{l}1,49 \mathrm{Ac} \\
0,84 \mathrm{Bb}\end{array}$ & $\begin{array}{l}2,09 \mathrm{Ab} \\
1,09 \mathrm{Bb}\end{array}$ & $\begin{array}{l}2,71 \mathrm{Aa} \\
1,54 \mathrm{Ba}\end{array}$ \\
\hline & \multicolumn{5}{|c|}{ Zn-óxidos de $\mathrm{Mn}, \mathrm{mg} \mathrm{dm}^{-3}$} \\
\hline $\begin{array}{r}30 \\
150\end{array}$ & $\begin{array}{l}0,61 \mathrm{Ae} \\
0,40 \mathrm{Ac}\end{array}$ & $\begin{array}{l}1,27 \mathrm{Ad} \\
0,64 \mathrm{Bbc}\end{array}$ & $\begin{array}{l}1,86 \mathrm{Ac} \\
0,91 \mathrm{Bb}\end{array}$ & $\begin{array}{l}2,66 \mathrm{Ab} \\
1,27 \mathrm{Ba}\end{array}$ & $\begin{array}{l}3,32 \mathrm{Aa} \\
1,60 \mathrm{Ba}\end{array}$ \\
\hline
\end{tabular}

(1) Letras maiúsculas não diferem entre si, na coluna (épocas), e letras minúsculas não diferem entre si, na linha (doses), pelo teste de Tukey a $5 \%$, para cada fração.

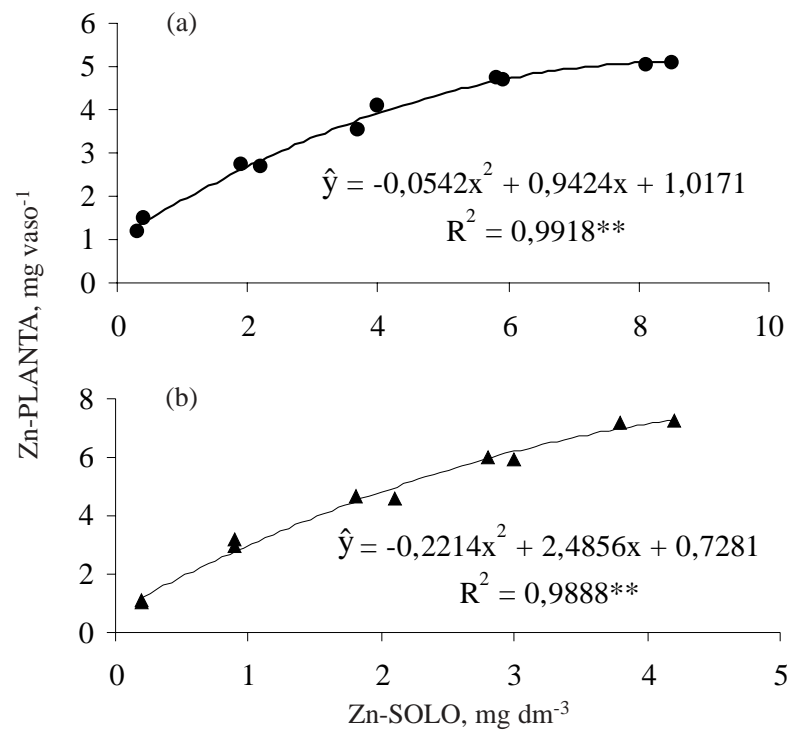

Figura 1. Relação entre teor de zinco extraído por DTPA e na parte aérea da planta em amostras coletadas: (a) antes do plantio (solo) e depois do $1^{\circ}$ corte (planta) e (b) depois do $3^{\circ}$ corte (solo e planta), em dois níveis de calagem.

verificar (Figuras 3 e 5) quais frações do Zn no solo estariam efetivamente disponíveis.

As relações apresentadas nas figuras 2 e 3 indicam que todas as frações contribuíram para o Zn disponível, com exceção da fração ligada aos óxidos de Fe, no tratamento com calagem ( $\mathrm{V}=70 \%$ ) antes do plantio de tifton, para a qual a regressão não foi significativa. As figuras 4 e 5 confirmam que o Zn considerado disponível encontra-se principalmente na fração trocável e na associada aos óxidos de $\mathrm{Mn}$ e à matéria orgânica, exceção feita aos tratamentos sem calagem ( $\mathrm{V}=42 \%)$, no qual a regressão foi significativa também para o Zn ligado aos óxidos de Fe. Desse modo, o Zn considerado disponível está associado principal mente às frações de mais fácil solubilização e, ou, mineralização. Alvarez et al. (1996) encontraram correlações significativas entre o Zn absorvido pela planta e os teores do elemento nas frações: solúvel em água + trocável, compl exado organicamente e ligado aos óxidos de Fe amorfos, e não-significativas para o Zn ligado aos óxidos de F e cristalinos e o residual.

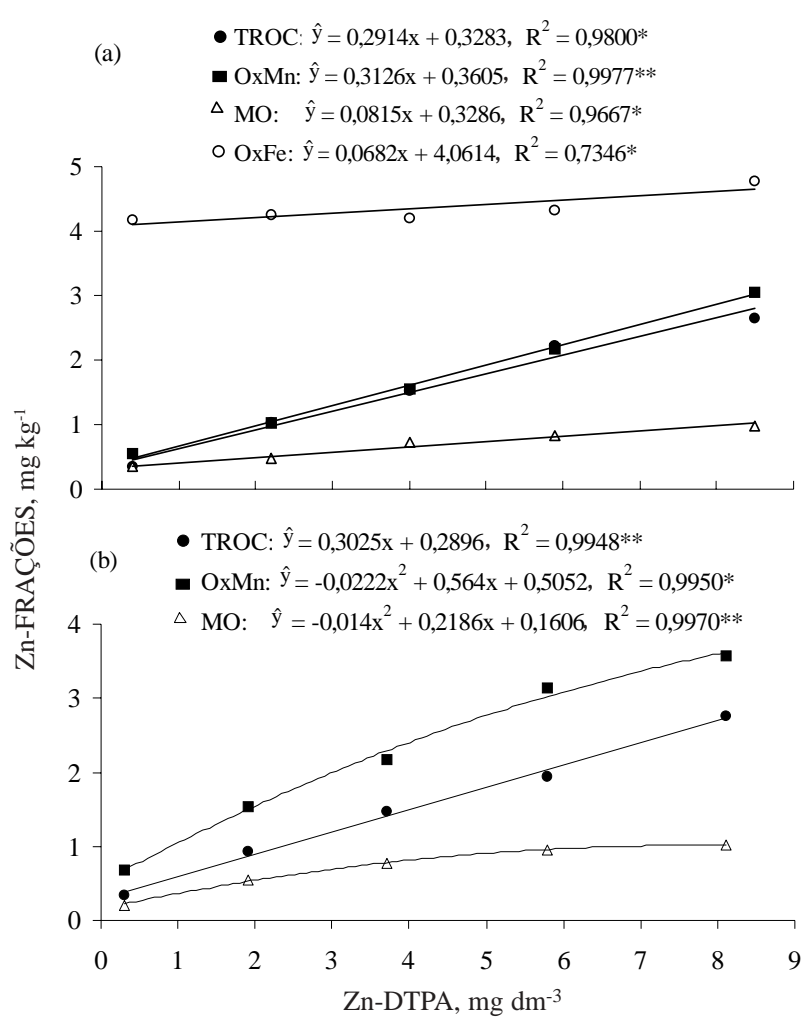

Figura 2. Relação entre as frações de Zn e o extraído por DTPA em amostras de solo sem calagem (a) e com calagem (b), coletadas antes do plantio. 


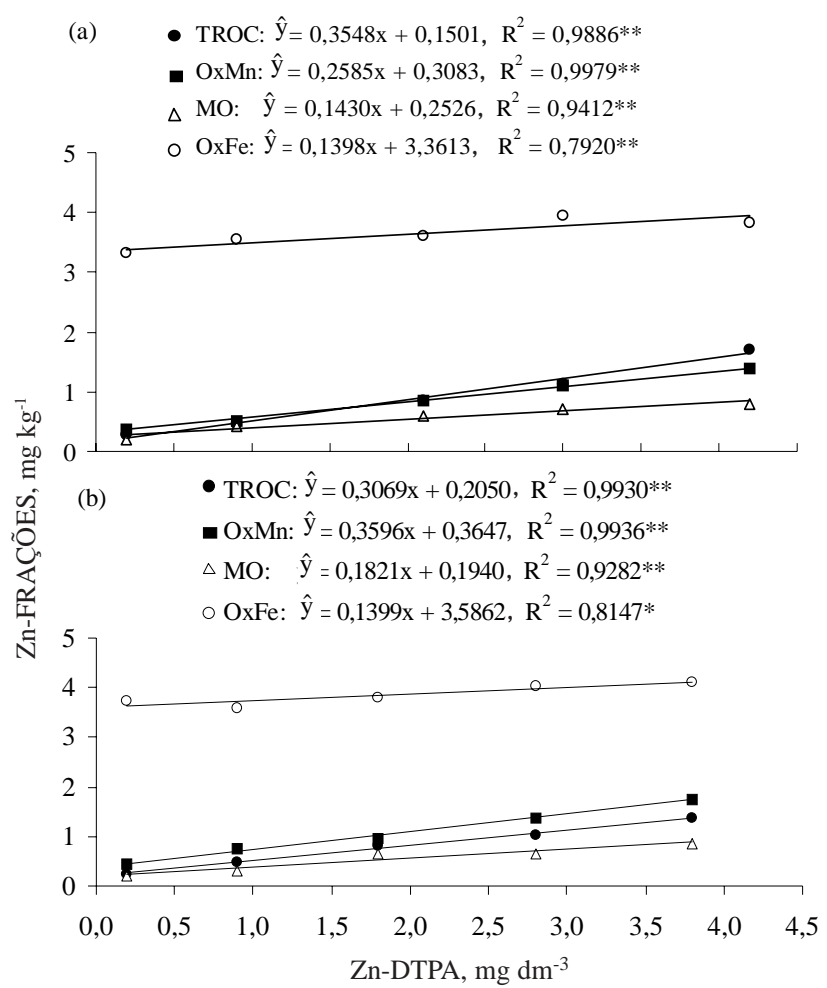

Figura 3. Relação entre as frações de zinco e o extraído por DTPA em amostras de solo sem calagem (a) e com calagem (b), coletadas depois do $3^{\circ}$ corte.

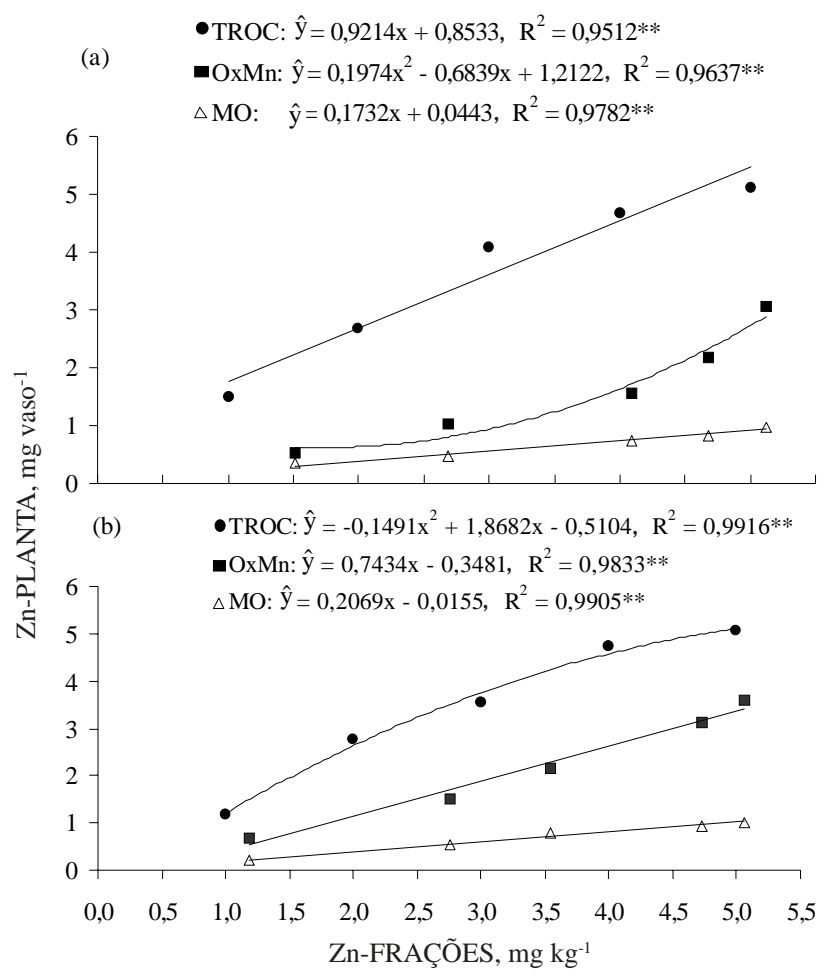

Figura 4. Relação entre o zinco na parte aérea da planta ( $1^{\circ}$ corte) e as frações de zinco em amostras de solo sem calagem (a) e com calagem (b), coletadas antes do plantio.

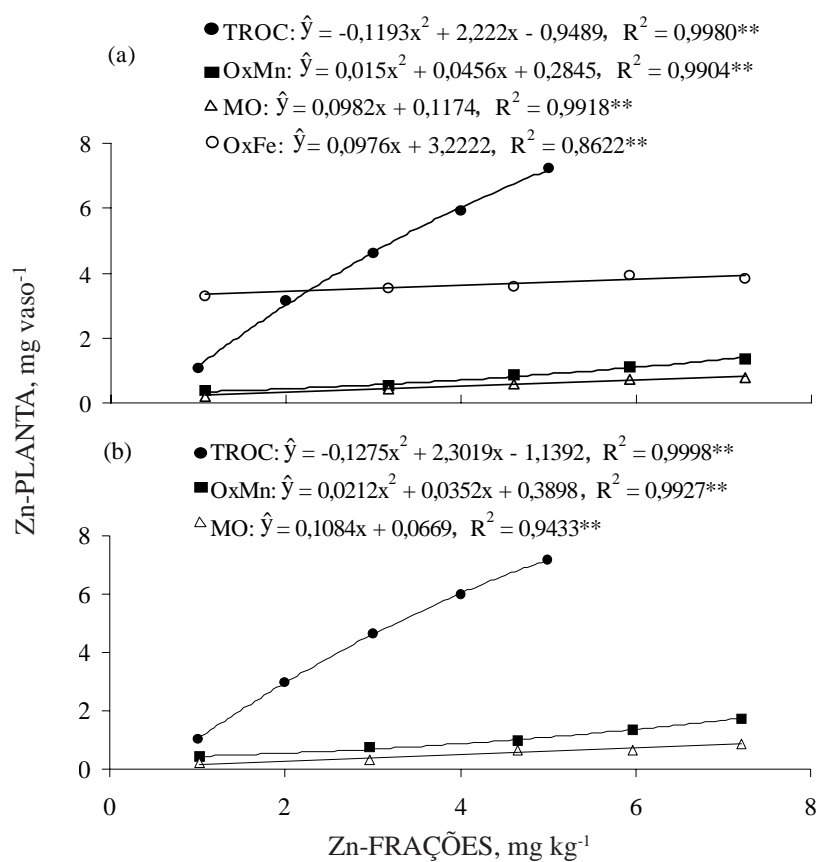

Figura 5. Relação entre o zinco na parte aérea da planta e as frações de zinco em amostras de solo sem calagem (a) e com calagem (b), coletadas depois do $3^{\circ}$ corte.

Segundo Ribeiro Filho et al. (2001), as correlações entre as frações dos metais no solo e suas concentrações na parte aérea são importantes para se tentar entender a relação entre formas químicas no sol o e biodisponi bilidade ou absorção pela planta. Entretanto, tais autores somente obtiveram correlação significativa entre o teor de zinco na planta e aquele presente na fração trocável, além do extraído por DTPA.

\section{CONCLUSÕES}

1. A distribuição de Zn entre as frações do solo, independentemente da época de amostragem, foi: residual >ligado aos óxidos deFe >ligado aos óxidos de $M n$ >trocável > ligado à matéria orgânica.

2. Tanto o Zn-DTPA quanto o Zn nas frações: trocável, ligado à matéria orgânica e aos óxidos de $\mathrm{Mn}$ foram significativamente relacionados com o Zn absorvido pela planta e, por isso, representaram eficientemente a disponi bilidade do el emento.

\section{LITE RATURA CITADA}

ABREU, C.A. \& RAIJ , B. van. Efeito da reação do solo no zinco extraído pelas soluções de DTPA e Mehlich-1. Bragantia, 55:357-363, 1996. 
ABREU, C.A.; FERREIRA, M.E. \& BORKERT, C.M. Disponibilidade e avaliação de elementos catiônicos: zinco e cobre. In: FERREIRA, M.E.; CRUZ, M.C.P.; RAIJ , B. van $\&$ ABREU, C.A., eds. Micronutrientes e elementos tóxicos na agricultura. J aboticabal, CNPq/FAPESP/POTAFOS, 2001. p.125-141.

ALVAREZ,J .M.; OBRADOR, A. \& RICO, M.I. Effects of chelated zinc, soluble and coated fertilizers, on soil zinc status and zinc nutrition of maize. Comm. Soil Sci. Plant Anal., 27:719, 1996.

AMACHER, M.C. Nickel, cadmium and lead. In: SPARKS, D.L.; PAGE, A.L.; HELMKE, P.A.; LOEPPERT, R.H.; SOLTANPOUR, P.N.; TABATABAI, M.A.; J OHNSTON, C.T. \& SUMMER, M.E., eds. Methods of soil analysis: Chemical methods, part 3. Madison, Soil Science Society of America, 1996. p.739-768.

AMARAL SOBRINHO, N.M.B.; VELLOSO, A.C.X. \& COSTA, L.M. Simulação de aterro de resíduos siderúrgicos: movimento de metais pesados em colunas de solo. In: CONGRESSO BRASILEIRO DE CIÊNCIA DO SOLO, 21., Goiânia, 1993. Resumos. III. Sociedade Brasileira de Ciência do Solo, p.295-296, 1993.

AMARAL SOBRINHO, N.M.B.; GOMES, M.F.; VELLOSO, A.C.X. \& OLIVEIRA, C. Fracionamento de zinco e chumbo em solos tratados com fertilizantes e corretivos. R. Bras. Ci. Solo, 21:17-21, 1997.

BATAGLIA, O.C.; FURLANI, A.M.C.; TEIXEIRA, J.P.F.; FURLANI, P.R. \& GALLO, J.R. Métodos de análise química de plantas. Campinas, Instituto Agronômico de Campinas, 1983. 48p. (Boletim Técnico, 78)

CAMARGO, O.A.; MONIZ, A.C.; J ORGE, J .A. \& VALADARES, J .M.A.S. Métodos de análise química, mineralógica efísica de solo do Instituto Agronômico de Campinas. Campinas, Instituto Agronômico de Campinas, 1986. 94p. (Boletim Técnico, 106)

CANTARELLA, H. \& RAIJ , B. van. Determinação de sulfato em solos. Método do $\mathrm{BaCl}_{2}$ em pó. Campinas, Instituto Agronômico de Campinas, 1997. 2p.

CAVALLARO, N. \& McBRIDE, M.B. Zinc and copper sorption and fixation by an acid soil clay: effect of selective dissolutions. Soil Sci. Soc. Am. J ., 48:1050-1054, 1984.

$\mathrm{CHAO}$, T.T. Selective dissolution of manganese oxides from soils and sediments with acidified hydroxilaminehydrochloride. Soil Sci. Soc. Am. Proc., 36:764-768, 1972.

CHOWDHURY, A.K.; MCLAREN, R.G.; CAMERON, K.C. \& SWIFT, R.S. Fractionation of zinc in some New Zealand soils. Comm. Soil Sci. Plant Anal., 28:301-312, 1997.

DAVIDSON, C.M.; THOMAS, R.P.; MCVEY, S.E.; PERALA, R.; LITTLEJ OHN, D. \& URE, A.M. Evaluation of a sequential extraction procedure for the speciation of heavy metals in sediments. Anal. Chim. Acta, 2914:277-286, 1994.

HOWARD, J .L. \& VANDENBRINK, W.J . Sequential extraction analysis of heavy metals in sediments of variable composition using nitriloacetic acid to counteract resorption. Environ. Pollut., 106:285-292, 1999.
LINDSAY, W.L. \& NORVELL, W.A. Development of a DTPA soil test for zinc, iron, manganese, and copper. Soil Sci. Soc. Am. J ., 42:421-428, 1978.

MA, Y.B. \& UREN, N.C. Transformations of heavy metals added to soil - aplication of a new sequential extraction procedure. Geoderma, 84:157-168, 1998.

MILLER, W.P.; MARTENS, D.C. \& ZELAZNY, L.W. Effect of sequence in extraction of trace metals from soils. Soil Sci. Soc. Am. J ., 50:598-601, 1986

OKAZAKI, M.; TAKAMIDOH, K. \& YAMANE, I. Adsorption of heavy metal cations on hydrated oxides and oxides of iron and aluminum with different crystallinities. Soil Sci. Plant Nutr., 32:523-533, 1986.

OLIVEIRA, M.F.G.; NOVAIS, R.F.; NEVES, J.C.L.; VASCONCELLOS, C.A. \& ALVES, V.M.C. Relação entre o zinco "disponível", por diferentes extratores, e as frações de zinco em amostras de solos. R. Bras. Ci. Solo, 23:827836, 1999.

RAIJ , B.van; QUAGGIO, J .A.; CANTARELLA, H.; FERREIRA, M.E.; LOPES, A.S. \& BATAGLIA, O.C. Análise química do solo para fins de fertilidade. Campinas, Fundação Cargill, 1987. 170p.

RIBEIRO FILHO, M.R.; SIQUEIRA, J .O.; CURI, N. \& SIMÃO, J.B.P. Fracionamento e biodisponibilidade de metais pesados em solo contaminado, incubado com materiais orgânicos einorgânicos. R. Bras. Ci. Solo, 25:495-507, 2001.

SHUMAN, L.M. Fractionation method for soil microelements. Soil Sci., 140:11-22, 1985.

SHUMAN, L.M. Effect of liming on the distribution of manganese, copper, iron, and zinc among soil fractions. Soil Sci. Soc. Am. J ., 50:1236-1240, 1986.

SPOSITO, G.; LUND, L.J. \& CHANG, A.C. Trace metal chemistry in arid-zone field soils amended with sewage sludge: I. Fractionation of $\mathrm{Ni}, \mathrm{Cu}, \mathrm{Zn}, \mathrm{Cd}$, and $\mathrm{Pb}$ in solid phases. Soil Sci. Soc. Am. J ., 46:260-264, 1982.

SIMS, J.T. Soil pH effects on the distribution and plant availability of manganese, copper and zinc. Soil Sci. Soc. Am. J ., 50:367-373, 1986.

SIMS, J.T. \& KLINE, J.S. Chemical fractionation and plant uptake of heavy metals in soils amended with co-composted sewage sludge. J . Environ. Qual., 20:387-395, 1991.

STAHL, R.S. \& J AMES, B.R. Zinc sorption by iron-oxide-coated sand as a function of pH. Soil Sci. Soc. Am. J ., 55:12871290, 1991a.

STAHL, R.S. \& J AMES, B.R. Zinc sorption by manganese-oxidecoated sand as a function of pH. Soil Sci. Soc. Am. J., 55:1291-1294, 1991b.

TESSIER, A.; CAMPBELL, P.G.C. \& BISSON, M. Sequential extraction procedure for the speciation of particulate trace metals. Anal. Chem., 51:844-851, 1979.

ZHANG, M.; ALVA, A.K.; LI, Y.C. \& CALVERT, D.V. Chemical association of $\mathrm{Cu}, \mathrm{Zn}, \mathrm{Mn}$ and $\mathrm{Pb}$ in selected sandy citrus soils. Soil Sci., 162:181-188, 1997.

ZHU, B. \& ALVA, A.K. Distribution of trace metals in some sandy soils under citrus production. Soil Sci. Soc. Am. J ., 57:350-355, 1993. 